\title{
Disentangling the Cosmic Web with Lagrangian Submanifold
}

\author{
Sergei F. Shandarin and Mikhail V. Medvedev
}

Department of Physics and Astronomy, University of Kansas, Lawrence, KS 66045

\begin{abstract}
The Cosmic Web is a complicated highly-entangled geometrical object. Remarkably it has formed from practically Gaussian initial conditions, which may be regarded as the simplest departure from exactly uniform universe in purely deterministic mapping. The full complexity of the web is revealed neither in configuration no velocity spaces considered separately. It can be fully appreciated only in six-dimensional (6D) phase space. However, studies of the phase space is complicated by the fact that every projection of it on a three-dimensional (3D) space is multivalued and contained caustics. In addition phase space is not a metric space that complicates studies of geometry. We suggest to use Lagrangian submanifold i.e., $\mathbf{x}=\mathbf{x}(\mathbf{q})$, where both $\mathbf{x}$ and $\mathbf{q}$ are $3 \mathrm{D}$ vectors instead of the phase space for studies the complexity of cosmic web in cosmological $N$-body dark matter simulations. Being fully equivalent in dynamical sense to the phase space it has an advantage of being a single valued and also metric space.
\end{abstract}

Keywords. voids, dark matter, N-body simulations, excursion set, large-scale structure

\section{Introduction}

Modern redshift surveys reveal an intricate 3D structure in the spatial distribution of galaxies, often called a Cosmic Web. Generic building blocks of the large-scale structure (LSS) are: halos, filaments, walls, and voids. Historically, halos have attracted the most of attention in theoretical studies of the LSS formation. From the observational point of view, halos are most closely related to galaxies, galaxy groups and clusters of galaxies, which provide most of information about our universe. However, direct modeling of galaxy formation based on fundamental laws of physics is precluded by enormous complexity of the physical processes involved, such as the highly nonlinear gravitational evolution of collisionless dark matter (DM) together with the hydrodynamical and thermal processes in baryons including star formation and the stellar wind feedback, shocks and supernovae explosions, gas accretion onto black holes in active galactic nuclei and the feedback via relativistic jets, and others. Hence various semi-empirical models of galaxy formation have been suggested, e.g., that galaxies are formed in the host DM halos of corresponding masses. The DM halos themselves are formed in a chain of mergers of smaller DM halos which may start from tiny halos of a planet mass (Diemand, et al. 2005). When two or more halos merge their remnants may survive for a long time as subhalos and/or streams within the resultant halo. Therefore, DM halos are likely to have a hierarchical structure resembling a Russian doll or "Matryoshka", where each subhalo includes a number of even smaller subhalos down to the smallest halos allowed by the initial power spectrum (Diemand, et al. 2005; Ghigna, et al. 1998).

In cosmological $N$-body simulations, DM halos were loosely defined as compact concentrations of the simulation particles in configuration space. Numerous sophisticated methods to identify halos, subhalos and other elements of the LSS have been developed over decades (see e.g. Shandarin (1983); Davis, et al. (1985); Vogelsberger \& White (2011); Knebe, et al. (2013); Hoffmann, et al. (2014) and references therein). Most of 

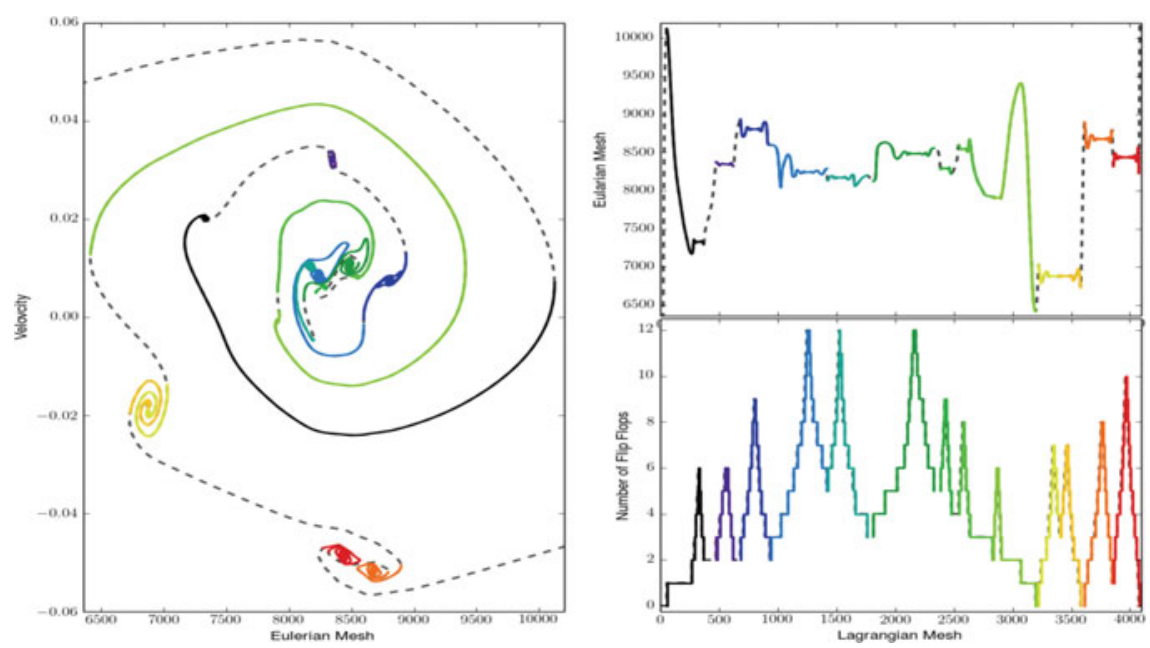

Figure 1. (left panel) The phase space of a one-dimensional halo simulated from random but smooth initial condition. The individual subhalos are shown by different colors. (right panel) Fields $x(q)$ and $n_{\mathrm{ff}}(q)$ are plotted in the top and bottom panels respectively.

the methods use only the configuration space information. However, they suffer from the projection effect that causes dynamically distinct structures in phase space to overlap in configuration space (for illustration, see the left panel of Fig. 1). Unfortunately, using all dynamical information provided by phase space is complicated by the fact that it is not a metric space (Ascasibar \& Binney 2005). Here we propose an novel technique which does not suffer from all the above problems.

\section{Lagrange Submanifold}

We propose to identify the elements of the LSS by analyzing the mapping — the epimorphism - namely, $\mathbf{x}=\mathbf{x}(\mathbf{q}, t)$, where $\mathbf{x}$ and $\mathbf{q}$ are the coordinates of the particles in Eulerian and Lagrangian spaces respectively, (Shandarin \& Medvedev 2012). Topologically, this mapping, referred to as the Lagrangian submanifold, is a $3 \mathrm{D}$ sheet in the the six-dimensional $(\mathbf{q}, \mathbf{x})$ space. Our method is based on a concept of a DM sheet $\mathbf{v}=\mathbf{v}(\mathbf{x}, t)$, which major difference from the conventional interpretation of $N$-body simulations is in a different role of simulation particles. In contrast to the common interpretation of particles as carriers of mass, the new approach treats them as massless markers of the vertices in a tessellation of the $3 \mathrm{D}$ DM sheet in $6 \mathrm{D}$ phase space. The particles' mass is uniformly distributed inside each tetrahedra of the tessellation (Shandarin, et al. 2012; Abel, et al. 2012). Once the tessellation is built in the initial state of the simulation, it must remain intact through the whole evolution because of the Liouville's theorem, as long as the thermal velocities of the DM particles are vanishing. This requirement differs markedly from the Delaunay tessellation approach proposed by Schaap \& van de Weygaert (2000) for estimating the density from particle distributions.

We stress that whereas both $(\mathbf{x}, \mathbf{v})$ and $(\mathbf{q}, \mathbf{x})$ spaces contain all the information about a dynamical system, the latter is a metric space and hence superior to the non-metric phase space. Moreover, the Lagrangian submanifold mapping, $\mathbf{x}=\mathbf{x}(\mathbf{q})$, is a single-valued function, unlike the phase-space mappings $\mathbf{v}=\mathbf{v}(\mathbf{x})$ or $\mathbf{x}=\mathbf{x}(\mathbf{v})$ which are multivalued.

To illustrate the above statements, Fig. 1 shows the phase space of a halo evolved in a one-dimensional (1D) universe from some smooth random initial condition. A halo can be naturally defined as the region in Eulerian space where the number of streams is greater 


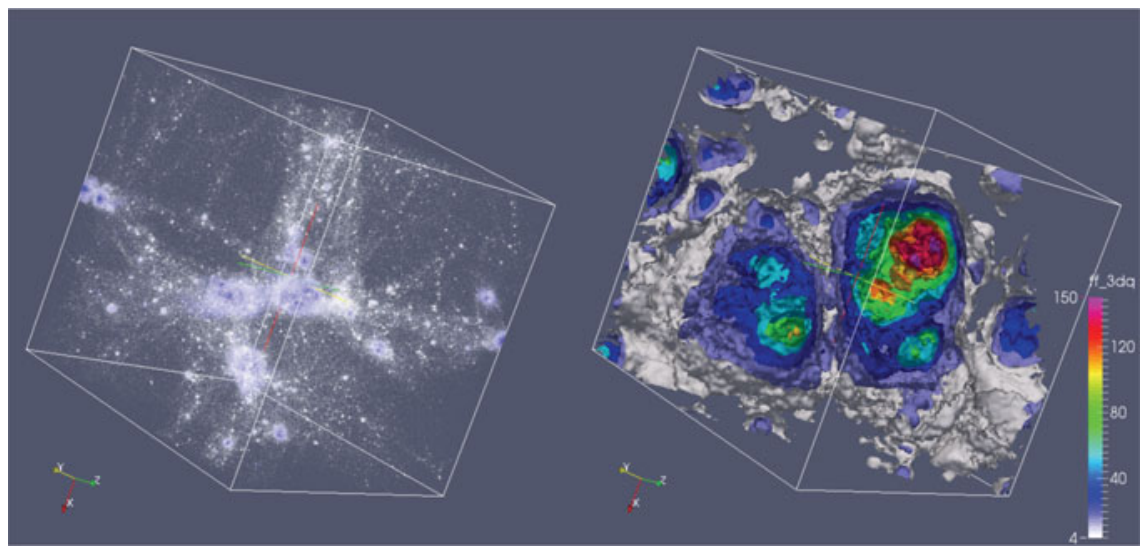

Figure 2. (left panel) The scatter plot of the structure in Euler space, $\mathbf{x}$, in the simulation of 1 $\mathrm{Mpc} / h$ box in the $\Lambda \mathrm{CDM}$ cosmology at $z=0$. The colors from white to red correspond to the range $n_{\mathrm{ff}} \geqslant 4$. (right panel) The contour plot of $n_{\mathrm{ff}}(\mathbf{q})$ field in the Lagrange space, $\mathbf{q}$, cut with a $2 \mathrm{D}$ cross-section plane through the center of the simulation cube. The same colors are is used.

than one. The number of stream changes by two at caustics where the tangent to the phase space curve becomes vertical and the density in the corresponding stream becomes formally infinite. Subhalos and streams are shown by different colors. It is obvious from the figure that identifying individual subhalos in the configuration (Eulerian) space is difficult even in a simple 1D model due to projection effects and the presence of tidal streams. The situation with $3 \mathrm{D}$ simulations is even more complex.

In our approach, the initial (Lagrangian) coordinates $q_{\mathrm{i}}$ of the particles, which are in essence their IDs (which are known exactly, by the way), increase monotonically. However, their final (Eulerian) coordinates $x_{\mathrm{i}}$ are not monotonic, see top-right panel of Fig. 1, i.e., there are DM fluid elements with $x_{\mathrm{i}+1}<x_{\mathrm{i}}$ while $q_{\mathrm{i}+1}>q_{\mathrm{i}}$. We call a swap of the Eulerian coordinates of the two neighboring Lagrange particles as a flip-flop. In 3D, this corresponds to a formal change of the sign of the Jacobian $J(\mathbf{q}, t)=\left|\partial x_{i} / \partial q_{j}\right|$. The total number of flip-flops at a given time is shown in the bottom panel of Fig. 1. Colors show individual peaks of the flip-flop field in Lagrangian coordinates. The correspondence of the flip-flop peaks in Lagrangian space to the individual subhalos in the phase space is striking. The tidal streams and their progenitor halos are also easily, unambiguously and robustly identified via the flip-flop field, see the bottom panel of Fig. 1.

\section{Implications}

Now, we demonstrate our technique in real DM-only $N$-body simulations using an appropriately modified GADGET code (Springel 2005). The initial conditions were generated with $\mathrm{N}$-GenIC code with the standard $\Lambda \mathrm{CDM}$ cosmology, $\Omega_{m}=0.3, \Omega_{\Lambda}=0.7, \Omega_{b}=$ $0, \sigma_{8}=0.9, h=0.7$ and the initial redshift $z=50$. For illustration purposes, we show here a small zoomed-in simulation with $256^{3}$ DM particles in a $1 h^{-1} \mathrm{Mpc}$ (comoving) box. The the main purpose of this example is to demonstrate that the flip-flop field of halos in a highly nonlinear dynamic state, which still retains rich information about the substructure in haloes.

Fig. 2 (left panel) shows the map of the excursion set $n_{\mathrm{ff}}(\mathbf{q}, z=0) \geqslant 4$ to Eulerian space and the flip-flop field in Lagrangian space. The colors - white-blue-green-red - of the particles represent the number of flip-flops. Fig. 2 (right panel) shows the corresponding flip-flop field in Lagrangian space with the same color coding. Obviously, 

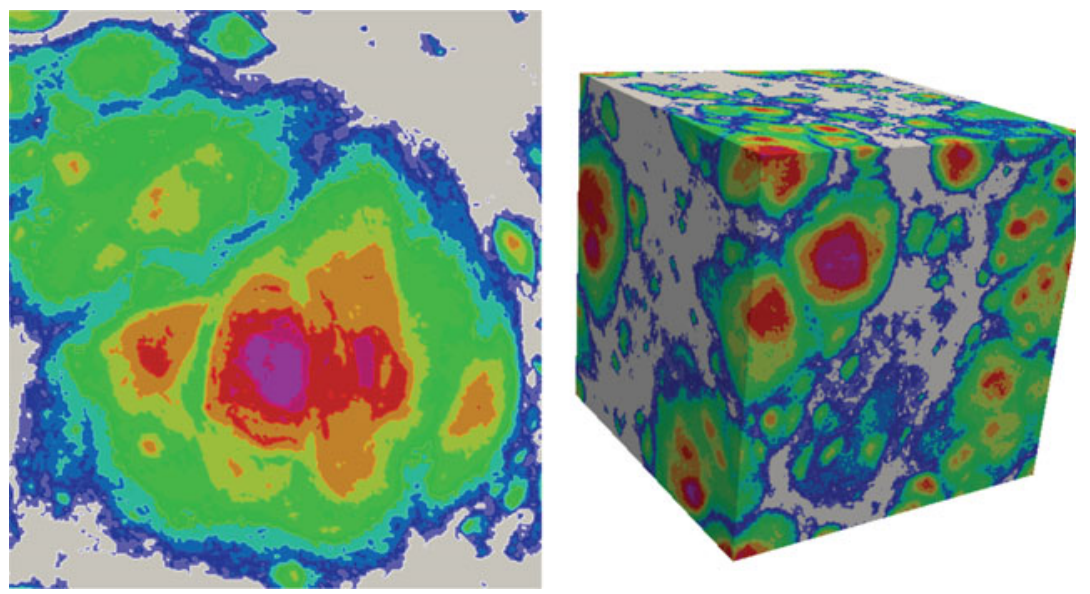

Figure 3. The contour plot of $n_{\mathrm{ff}}$ (q) field, i.e., the number of flip-flops in the Lagrange space, for the same simulation shown in Fig. 2. Right panel show the entire simulation cube and the left panel show one of six faces of the cube. Much finer contour levels demonstrate the complex "Matrioshka"-type structure of halos/subhalos and of the entire Cosmic Web.

this flip-flop field in Lagrangian space traces the distribution of matter in the universe extremely accurately. One can clearly see that halos (and the LSS, in general) form a large number of distinct flip-flop peaks in Lagrangian space. In order to reveal the much greater richness and complexity of the structure of LSS in the flip-flop field, we also plot a full $3 \mathrm{D}$ cube and its one face in the Lagrangian space in Fig. 3. This figure shows a complex hierarchy of peaks in much greater detail. We stress that the regions in Lagrange space with zero flip-flops are, by definition, voids. Thus, the flip-flop field $n_{\mathrm{ff}}(\mathbf{q}, z)$, along with the number of streams field $n_{\mathrm{str}}(\mathbf{x}, z)$ (Shandarin, et al. 2012) are the superior void detectors in $N$-body simulations, as they do not suffer from the poor density contrast or other issues. Note, it differs from another useful "sOrigami" method (Neyrinck 2012), as our method does not contain any free parameters. Our method is also "Diophantine" as it deals with integer numbers of flip-flops, so it does not suffer from numerical accuracy errors. Therefore, we can call our universal 'flip-flop' approach to cosmological LSS formation the "Diophantine cosmology".

\section{References}

T. Abel, O. Hahn, \& R. Kaehler, MNRAS 427, 61 (2012).

Y. Ascasibar \& J. Binney, MNRAS 356, 872 (2005).

M. Davis, G. Efstathiou, C. S. Frenk, \& S. D. M. White, ApJ 292, 371 (1985).

J. Diemand, B. Moore, \& J. Stadel, Nature 433, 389 (2005).

S. Ghigna et al., MNRAS 300, 146 (1998).

K. Hoffmann et al., MNRAS 442, 1197 (2014).

A. Knebe et al., MNRAS 435, 1618 (2013).

M. C. Neyrinck, MNRAS 427, 494 (2012).

W. E. Schaap \& R. van de Weygaert, A\&A 363, L29 (2000).

S. F. Shandarin, Soviet Astronomy Letters 9, 104 (1983).

S. F. Shandarin, S. Habib, \& K. Heitmann, Phys. Rev. D 385, 083005 (2012).

S. F. Shandarin \& M. V. Medvedev, submitted; ArXiv:1409.7634 (2014).

V. Springel, MNRAS 364, 1105 (2005).

M. Vogelsberger \& S. D. M. White, MNRAS 413, 1419 (2011). 